\title{
Impacto da pandemia por COVID-19 em pacientes com transtornos alimentares: considerações para profissionais de saúde mental
}

\author{
Impact of COVID-19 pandemic on patients with eating disorders: considerations for \\ mental health professionals
}

\section{Impacto de la pandemia de COVID-19 en pacientes con trastornos alimentarios: consideraciones para profesionales de salud mental}

Felipe Alckmin-Carvalho $\mathbb{D}^{\mathrm{a}}$

a Instituto de Psicologia da Universidade de São Paulo, Programa de Pós-Graduação em Psicologia Clínica - São Paulo/ SP - Brasil.

Instituição: Instituto de Psicologia da Universidade de São Paulo - São Paulo/SP - Brasil

DOI 10.5935/2318-0404.20210001

\section{Resumo}

Apresenta-se um manuscrito no formato de carta ao editor, em que são abordados os impactos da pandemia por COVID-19 na saúde mental de pessoas com transtornos alimentares. São discutidos os impactos mais prováveis, em termos de agravamento dos sintomas nos quadros de Anorexia Nervosa, Bulimia Nervosa e no Transtorno de Compulsão Alimentar. Por fim, são apresentadas considerações para o manejo clínico dos pacientes com a finalidade de mitigar os impactos da pandemia no comportamento alimentar.

Palavras-chaves: COVID-19; Infecções por Coronavirus; Saúde Mental; Comportamento Alimentar; Transtornos da Alimentação e da Ingestão de Alimentos; Psicoterapia

\section{Abstract}

A manuscript in the form of a letter to the editor is presented, in which the impacts of COVID-19 pandemic on the mental health of people with eating disorders are addressed. The most likely impacts are discussed, in terms of worsening of symptoms in Anorexia Nervosa, Bulimia Nervosa and Binge Eating Disorder. Finally, 
considerations are presented for the clinical approach of patients in order to mitigate the impacts of pandemics on eating behavior.

Keywords: COVID-19; Coronavirus Infections; Mental Health; Feeding Behavior; Feeding and Eating Disorders; Psychotherapy

\section{Resumen}

Se presenta un manuscrito en forma de una carta al editor, en el que se abordan los impactos de la pandemia de COVID-19 sobre la salud mental de las personas con transtornos alimentarios. Se discuten los impactos más probables, en términos de empeoramiento de los síntomas en la Anorexia Nervosa, la Bulimia Nerviosa y en el Transtorno de la Compulsión Alimentaria. Finalmente, se presentan consideraciones para el manejo clínico de los pacientes a fin de mitigar los impactos de la pandemia en el comportamiento alimentario.

Palabras clave: COVID-19; Infecciones por Coronavirus; Salud Mental; Conducta Alimentaria; Trastornos de Alimentación y de la Ingestión de Alimentos; Psicoterapia

Desde a primeira morte por COVID-19 no Brasil, registrada em fevereiro de 2020, a pandemia já matou, em doze meses, mais de 200.000 pessoas. Infectou pelo menos outras 7 milhões, no país, sendo essa prevalência possivelmente subestimada. A instabilidade política e a ineficiência da resposta ao COVID-19 no Brasil têm agravado essa crise sanitária sem precedentes ${ }^{1}$. Discursos e comportamentos inconsistentes do presidente da república, que, na contramão das orientações da Organização Mundial da Saúde (OMS), desencoraja abertamente as sensatas medidas de distanciamento social, agravam a sensação de insegurança e produzem medo e ansiedade em níveis alarmantes ${ }^{1}$. As principais preocupações e medos da população têm relação com a infecção pela COVID-19, a transmissão do vírus, o adoecimento e a morte própria ou de entes queridos, o caos econômico e político.

Em situações adversas como a atual, pessoas com Transtornos Alimentares (TA) tendem a ter seus sintomas agravados ${ }^{2}$. Indivíduos com sintomas subclínicos podem passar a preencher os critérios para o diagnóstico de um TA e pacientes que já estavam em fase de remissão podem voltar a apresentar padrões de alimentação sintomáticos, com sofrimento clinicamente significativo associado.

Algumas tendências podem ser previstas, a partir do perfil cognitivo e comportamental associado a cada TA, verificado em pesquisas empíricas e a partir da experiência clínica. Pesquisadores indicam que pacientes com Anorexia Nervosa costumam apresentar dificuldade de lidar com situações de mudanças, risco, incerteza ou falta de controle ${ }^{3}$. Frente à sensação de incontrolabilidade é possível que esses pacientes voltem a controlar seu peso e mediadas corporais, e a se engajarem em comportamentos para perder peso, com função de aliviar a sensação de vulnerabilidade.

Pacientes com Transtorno de Compulsão Alimentar e Bulimia Nervosa, por sua vez, que tendem a apresentar "comer emocional" ${ }^{4}$, podem fazer uso da comida para diminuir sensações de solidão, frustração 
e tédio, decorrentes das medidas de restrição das atividades envolvendo relações humanas para conter as infecções por COVID19. Além disso, a necessidade de estocar certa quantidade de alimento para evitar a circulação nas cidades pode favorecer episódios de compulsão alimentar e de métodos compensatórios do ganho de peso.

Na Anorexia Nervosa, na Bulimia Nervosa e no Transtorno de Compulsão Alimentar verifica-se que a relação que se estabelece com a comida - restritiva ou excessiva, seguida ou não de métodos compensatórios de ganho de peso - tem função de autorregulação emocional| ${ }^{4,5}$. Adicionalmente, pacientes com esses TA costumam apresentar algum nível de alexitimia (dificuldade de identificar, descrever e comunicar sentimentos e de diferenciá-los de sensações corporais) ${ }^{6}$. Assim, frente à impossibilidade de falar sobre os sentimentos de modo adequado, aumenta a probabilidade de passagem ao ato (acting-out), por meio do uso da comida e do corpo para a expressão de sentimentos e sensações como angústia, vulnerabilidade, ansiedade e desesperança.

Ademais, entre pacientes com Bulimia Nervosa e Compulsão Alimentar Periódica, sobretudo, as restrições de contato social e outros modos de privação associados às medidas preventivas da infecção por COVID-19 podem produzir sensação de isolamento, solidão e tristeza. Frente a essas sensações aversivas, o uso da comida como reforçador substituto da atenção social e afeto, operação comum nesses quadros ${ }^{7}$, tende a ocorrer. Portanto, expressivo ganho de peso em pacientes com Transtorno de Compulsão Alimentar e aumento da frequência dos episódios de compulsão e purgação entre indivíduos com Bulimia Nervosa devem ser monitorados e cuidados.

Nesse delicado e complexo cenário, clínicos que atendem a pessoas com TA devem estar atentos ao modo como cada paciente é afetado pela pandemia, considerando as particularidades de cada caso. As estratégias de tratamento devem contemplar o desenvolvimento de comportamentos alternativos com função de regulação emocional compatíveis com o contexto do paciente. Esses profissionais de saúde mental devem, ainda, estimular que seus pacientes coloquem em palavras os impactos objetivos e subjetivos decorrentes da pandemia. Devem também funcionar como ambiente que favoreça o desenvolvimento de estratégias para lidar com restrições e perdas que prescindam do uso da alimentação e do corpo para este fim.

Especialistas na área de TA têm recomendado uma série de medidas que podem reduzir os níveis de ansiedade e angústia desses pacientes durante a pandemia por COVID-192,7. Manter os atendimentos nutricionais, psicológicos e psiquiátricos por videoconferência tem se mostrado uma estratégia segura, viável e eficaz. Atualmente sabe-se que quanto maior o nível de exposição à cobertura da mídia da crise sanitária, mais elevados parecem permanecer os níveis de ansiedade e insegurança. Assim, tem-se recomendado a exposição a informações sobre a pandemia de modo controlado e monitorado.

Para manejar a solidão decorrente do isolamento social, encontros online são estimulados. Além disso, encontros presenciais, esporádicos, entre poucas pessoas, também em quarentena, em espaços abertos e ventilados, mantendo-se o distanciamento de aproximadamente dois metros e o uso de máscaras, podem ser importantes para manter a quarentena viável, em termos emocionais. Grupos de apoio online para pacientes com TA ou para a comunidade, de modo geral, também têm se mostrado uma alternativa interessante. 
Durante a pandemia por COVID-19 tem-se verificado o aumento do tempo de uso de redes sociais. Monitorar o tempo de exposição a essas redes e seus efeitos também é indicado, uma vez que exposição a essas redes costumam produzir efeitos negativos em termos de imagem corporal e autoestima entre pacientes com TA. Investir em atividades de lazer que não envolvam risco de contágio por COVID-19 também é uma medida estimulada. Para pacientes com TA que permanecem em casa, manter os horários das refeições e fazer as refeições em ambiente diferente de onde se trabalha ou dorme, quando possível, é recomendado.

Por fim, destaca-se que os comportamentos verificados entre pacientes com TA, como restrição alimentar severa, exercícios físicos em excesso, compulsão alimentar e purgação, quando perduram por longos períodos, podem produzir importante impacto fisiológico com potencial de imunossupressão ${ }^{8}$, condição associada a piores prognósticos em casos de infecção por Coronavírus ${ }^{9}$. Desse modo, possíveis infecções por COVID-19 entre pacientes com baixo peso, desnutrição severa e crônica, ou entre pacientes obesos, devem ser especialmente evitadas. Durante a pandemia de COVID-19 a continuidade dos atendimentos psicológicos, psiquiátricos e nutricionais são essenciais para preservar a saúde física e mental do paciente com transtorno alimentar e mitigar o impacto emocional da grave condição sanitária da atualidade.

Felipe Alckmin-Carvalho

\section{Referências}

1. Prado B. COVID-19 in Brazil: "So what?". Lancet. 2020; 395:1461.

2. Fernández-Aranda F, Casas M, Claes L, Bryan DC, Favaro A, Granero R. Menchón JM. COVID-19 and implications for eating disorders. European Eating Disorders Review: The Journal of the Eating Disorders Association. 2018; 28(3), 239.

3. Merwin RM, Timko CA, Moskovich AA, Ingle KK, Bulik CM, Zucker NL. Psychological inflexibility and symptom expression in anorexia nervosa. Eating Disorders. 2010; 19(1), 62-82.

4. Rotella F, Mannucci E, Gemignani S, Lazzeretti L, Fioravanti G, Ricca V. Emotional eating and temperamental traits in Eating Disorders: A dimensional approach. Psychiatry Research. 2018; 264, 1-8.

5. Brockmeyer T, Skunde M, Wu M, Bresslein E, Rudofsky G, Herzog W, Friederich HC. Difficulties in emotion regulation across the spectrum of eating disorders. Comprehensive Psychiatry. 2014; 55(3), 565-571.

6. Nowakowski ME, McFarlane T, Cassin S. Alexithymia and eating disorders: a critical review of the literature. Journal of Eating Disorders. 2013; 1(1), 21-36.

7. Vale AMOD \& Elias LR. Transtornos alimentares: uma perspectiva analítico-comportamental. Revista Brasileira de Terapia Comportamental e Cognitiva. 2011; 13(1), 52-70.

8. Cuesto G, Everaerts C, León LG, Acebes A. Molecular bases of anorexia nervosa, bulimia nervosa and binge eating disorder: Shedding light on the darkness. Journal of neurogenetics. 2017; 31 (4), 266-287.

9. Fernández-Aranda F, Casas M, Claes L, Bryan DC, Favaro A, Granero R \& Treasure J. COVID-19 and implications for eating disorders. European Eating Disorders Review. 2020; 28(3), 239-245.

Contribuições: Felipe Alckmin-Carvalho - Redação - Preparação do original. 


\section{Correspondência}

\section{Felipe Alckmin-Carvalho}

felipealckminc@gmail.com / E-mail alternativo: felipcarvalho@usp.br

Submetido em: 18/05/2020

Aceito em: 19/04/2021 